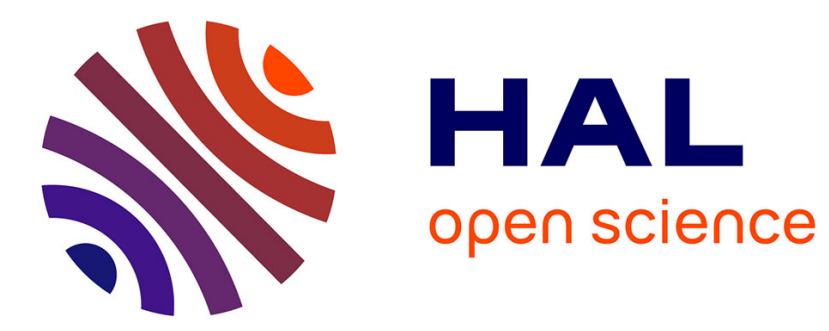

\title{
Design of a variable frequency comb reflectometer system for the ASDEX Upgrade tokamak
}

T. Happel, W. Kasparek, Pascale Hennequin, K. Höfler, Cyrille Honoré, Asdex Upgrade Team

\section{- To cite this version:}

T. Happel, W. Kasparek, Pascale Hennequin, K. Höfler, Cyrille Honoré, et al.. Design of a variable frequency comb reflectometer system for the ASDEX Upgrade tokamak. Plasma Science and Technology, 2020, 22 (6), pp.064002. 10.1088/2058-6272/ab618c . hal-02571938

\section{HAL Id: hal-02571938 \\ https://hal.science/hal-02571938}

Submitted on 7 Jan 2021

HAL is a multi-disciplinary open access archive for the deposit and dissemination of scientific research documents, whether they are published or not. The documents may come from teaching and research institutions in France or abroad, or from public or private research centers.
L'archive ouverte pluridisciplinaire HAL, est destinée au dépôt et à la diffusion de documents scientifiques de niveau recherche, publiés ou non, émanant des établissements d'enseignement et de recherche français ou étrangers, des laboratoires publics ou privés. 


\title{
Design of a Variable Frequency Comb Reflectometer System for the ASDEX Upgrade Tokamak
}

\author{
T. Happel, ${ }^{1, *}$ W. Kasparek, ${ }^{2}$ P. Hennequin, ${ }^{3}$ K. Höfler, ${ }^{1,4}$ C. Honore,${ }^{3}$ and the ASDEX Upgrade Team ${ }^{5}$ \\ ${ }^{1}$ Max-Planck-Institut für Plasmaphysik, Boltzmannstr. 2, 85748 Garching, Germany \\ ${ }^{2}$ IGVP, Universität Stuttgart, Pfaffenwaldring 31, 70569 Stuttgart, Germany \\ ${ }^{3}$ Laboratoire de Physique des Plasmas, Ecole Polytechnique, 91128 Palaiseau, France \\ ${ }^{4}$ Physik-Department E28, Technische Universität München, James-Franck-Str. 1, 85748 Garching, Germany \\ ${ }^{5}$ see the author list "H. Meyer et al., Nucl. Fusion 59, 112014 (2019) (https://doi.org/10.1088/1741-4326/ab18b8)",
}

(Dated: 2019-11-15)

\begin{abstract}
Comb reflectometers offer the advantage of measuring at several radial positions in the plasma simultaneously. This allows the investigation of fast timescales during L-H transitions, I-phases, I-mode bursts, transients during heat wave propagation, etc. A drawback of many present-day systems is that they use a fixed frequency difference between the probing frequencies. Hence, although the central probing frequency can be varied, usually the probing frequency difference is fixed. The new design presented in this work uses an advanced microwave generation and detection scheme, which allows for arbitrary probing frequencies and probing frequency separations.
\end{abstract}

\section{INTRODUCTION}

A detailed understanding of physics processes in magnetic confinement fusion plasmas is of high importance in order to facilitate predictions for future devices, and possibly for their optimization in terms of efficiency. To obtain such a detailed understanding, detailed measurements of relevant physics quantities are required. Usually, not only good spatial and temporal resolution for a measurement is desired, but also that the measurement can be obtained at various radial locations in the plasmas at the same time. To this end, various diagnostic methods usually employ different lines of sight.

In the case of fluctuation reflectometry or Doppler backscattering, various radial positions can be probed by using different probing frequencies, since these reflect at different densities (O-mode) and additionally magnetic field strengths (X-mode). For fluctuation reflectometry and Doppler backscattering applications, usually the probing of different radial positions is obtained by using frequency hopping reflectometer systems [1-3]. These systems can change the probing frequency typically within less than a ms, which means that radial profiles of plasma parameters can usually obtained within $50-100 \mathrm{~ms}$, depending on the radial resolution and radial coverage chosen.

A somewhat different concept is based on the launch of several probing frequencies simultaneously into the plasma and their simultaneous detection [4-14]. In this way, the information of several radial positions is obtained at the same time. Several drawbacks have previously held these systems from becoming the standard of reflectometer probing techniques, which is still the hopping system. First, the probing frequency difference has so far mostly been constant (excluding Ref. [12]). Second, the detection part of such a reflectometer has to be significantly more advanced and complex than for a hopping system. Third, since a comb reflectometer requires the data of various probing frequencies acquired simultaneously, the data acquisition system is also a critical part of a

*Electronic address: tim.happel@ipp.mpg.de comb reflectometer.

This paper presents the design of a new comb reflectometer system for the W-band of frequencies $(75-110 \mathrm{GHz})$ to be built for use on the ASDEX Upgrade tokamak (AUG). A variable frequency difference will be implemented, enabling measurements at arbitrary radial separations. Furthermore, the center frequency of the comb can be moved over the whole Wband, resulting in a high flexibility of the system. Two emitter concepts are proposed, one is based on two-tone input into the frequency multiplier, while the other is based on three-tone input. While these concepts will probably yield somewhat less signal-to-noise ratios than the systems based on non-linear transmission lines $[5,7,9,11]$, they are expected to be significantly more flexible.

This paper is organized as follows: section II introduces the hardware concepts of two-tone ( II A) and three-tone ( II B) input into the multiplier, followed by the detection chain ( II C). In sec. III experimental measurement results are presented from both laboratory and an AUG plasma, followed by a summary in sec. IV.

\section{HARDWARE CONCEPTS}

Several methods exist for the generation of a comb of frequencies, of which the most common one is probably the usage of a so-called nonlinear transmission line (NLTL) [5, 7, $9,11]$. For the methods presented below, the nonlinear behavior of frequency multipliers is exploited. Therefore, the operational concept is briefly summarized.

Figure 1 shows the principle of frequency multiplication by the method of harmonic generation by a nonlinear element. A low frequency signal (here $14 \mathrm{GHz}$, blue in (a)) is connected to a nonlinear element, usually a diode. The spectrum of the input signal is depicted in fig.1(b), where only one peak at $14 \mathrm{GHz}$ is visible. The response of the nonlinear element (diode) can usually be written as $s(v)=i_{s}(\exp (\alpha v)-1)$, where $i_{s}$ and $\alpha$ are parameters representing the nonlinear character. The green curve in fig. 1(c) shows the diode output, which is close to zero unless the input signal (blue, (a)) is close to one. The spectrum of the diode output is shown in fig. 1(d). 

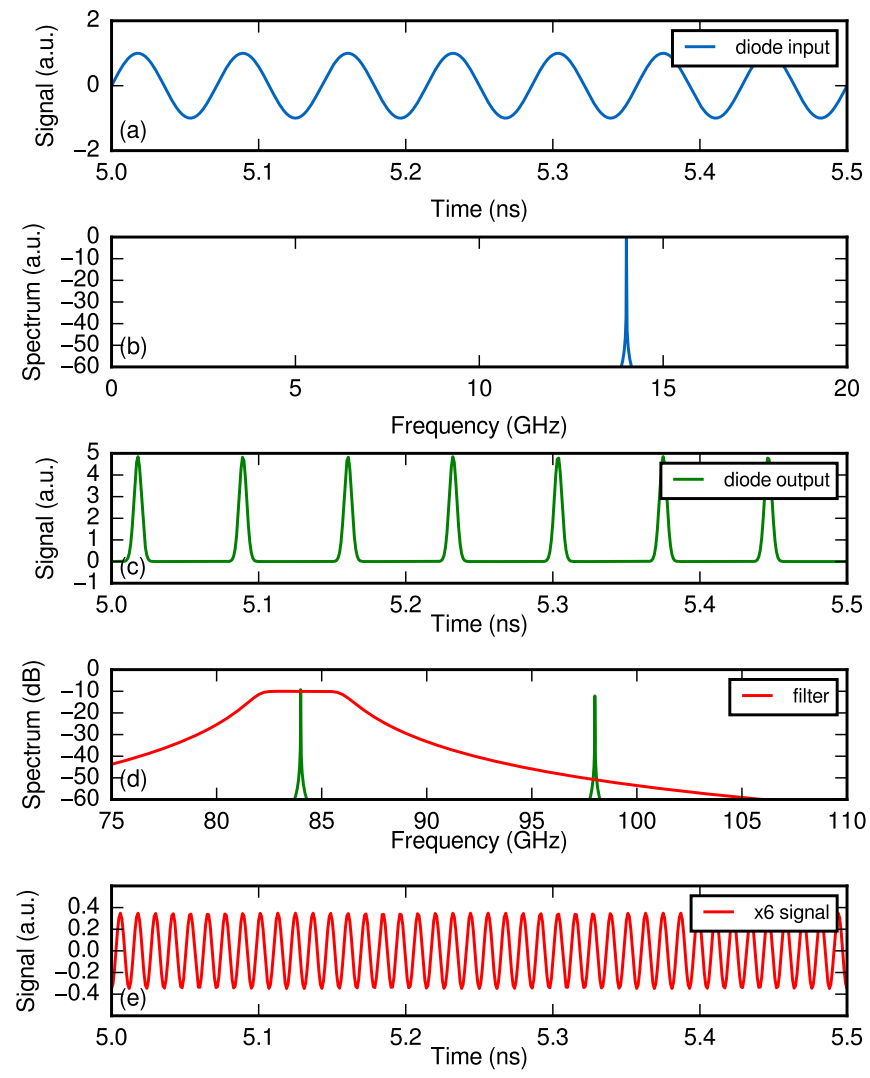

FIG. 1: Harmonic generation of a typical frequency multiplier. (a) Diode input signal, (b) its spectrum, (c) diode output, (d) its corresponding spectrum (green) and the filter (red) used to obtain the (e) multiplied signal.

Since the signal is not sinusoidal because of the nonlinearity, the specrum exhibits harmonics of the input frequency. Filtering the diode output in the appropriate frequency range in W-band and transforming back to time yields the red line in fig.1(e). The input frequency has been multiplied by six.

It should be noted that real multipliers are significantly more complex than this simple example. For instance, the filtering in W-band is obtained by building the multiplier such that several undesired harmonics are cancelled out internally. Nevertheless, this simple exercise is useful to be able to understand the mixer output investigated in the following.

\section{A. Two-tone Input to Multiplier}

In sec. II, a single frequency was used as input into the $\times 6$-multiplier. If two frequencies are used, $f_{1}$ and $f_{2}$, socalled intermodulation products will be generated on the output, which consist of $6 f_{1}, 5 f_{1}+f_{2}, 4 f_{1}+2 f_{2}, 3 f_{1}+3 f_{2}, 2 f_{1}+$ $4 f_{1}, f_{1}+5 f_{2}, 6 f_{2}$ This method has been explored in the context of Doppler backscattering previously on TCV [12].

In fig. 2 the result of using two frequencies $\left(f_{1}=14 \mathrm{GHz}\right.$, $\left.f_{2}=16 \mathrm{GHz}\right)$ as input into the multiplier is depicted. As before, fig. 2(a) shows an excerpt from the time trace of the diode input signal, and fig. 2(b) shows the corresponding spectrum.
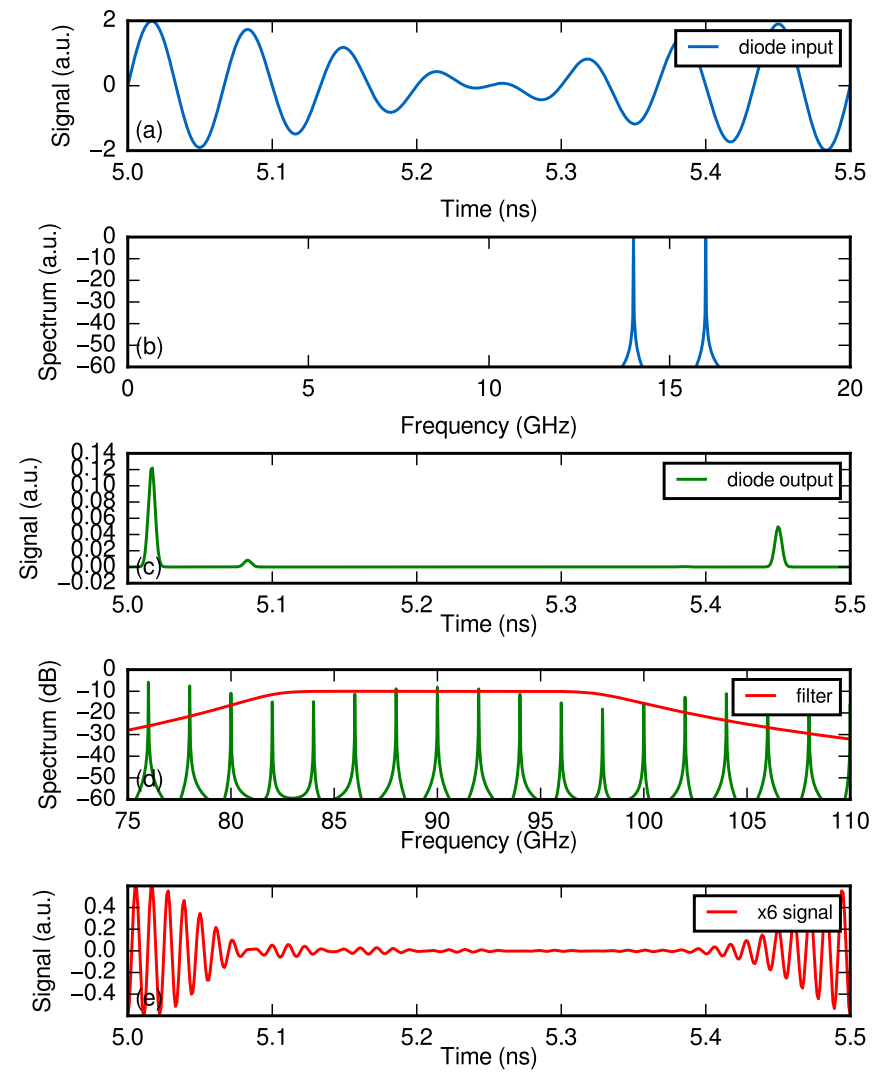

FIG. 2: Harmonic generation of a typical frequency multiplier using two-tone input. (a) Diode input signal, (b) its spectrum showing the two input frequencies at 14 and $16 \mathrm{GHz}$, (c) diode output, (d) its corresponding spectrum (green) and the filter (red) used to obtain the (e) multiplied signal. In (d), the comb of frequencies can be observed.

The beat frequency $f_{b}=f_{2}-f_{1}=2 \mathrm{GHz}$ is visible in the growth and the decay of the input signal (blue line) on a $0.5 \mathrm{~ns}$ timescale (period $T=1 /\left(f_{2}-f_{1}\right)$ ). In the spectrum, two peaks are visible. The output of the diode is again shown in green in fig. 2(c). In this case, the nonlinearity creates spikes of somewhat different shape than before. The spectrum of the signal after the diode (fig. 2(d)) shows again the harmonics and additionally the aforementioned intermodulation products. Specifically, and of importance for this application, there is a comb of frequencies in the W-band region between $6 f_{1}=84$ and $6 f_{2}=96 \mathrm{GHz}$, with the power strongest in the center, at $90 \mathrm{GHz}$. This will also be seen in the plasma tests later (cf sec. III C). As above, appropriate bandpass filtering will result in the desired frequency comb in the W-band range of frequencies. This concept has been tested both in the laboratory and on the ASDEX Upgrade tokamak, and the corresponding results are presented in secs. III A and III C.

\section{B. Three-tone Input to Multiplier}

The two-tone concept of sec. II A uses two synthesizers to generate the two frequencies. Similarly, one can develop a 
three-tone input to the multiplier. In this case, one can use a double sideband (DSB) mixer, and provide both LO $\left(f_{\mathrm{LO}}=\right.$ $12-18 \mathrm{GHz}$ ) and the desired difference frequency $f_{\mathrm{IF}}$. This will yield three frequencies on the RF port of the DSB mixer, $f_{\mathrm{LO}}$ and $f_{\mathrm{LO}} \pm f_{\mathrm{IF}}$. These can be provided to the $\times 6$-multiplier, which will yield a frequency comb on its output port. The comb will consist of $6 f_{\mathrm{LO}} \pm n f_{\mathrm{IF}}$, with $n=1 \ldots 3$. It should be noted that for this concept, the power of individual comb frequencies is particularly sensitive to relative input power levels. Hence, careful system calibration and configuration will be necessary to obtain optimum results across the full $W$-band of frequencies.

\section{Detection}

The various frequency components generated through either two-tone input (cf sec. II A) or three-tone input (cf sec. II B) will have to be detected, downconverted and fed into IQ-detectors for later data acquisition in a 14-channel $(7 \times$ inphase and quadrature signals) $20 \mathrm{MS} / \mathrm{s} 16$ bit system.

A simplified circuit diagram for the emission and detection part is shown in fig. 3. For simplicity, no amplifiers or attenuators are shown. Following sec. II A, the two-tone input into the multiplier is composed of $f_{1}=14 \mathrm{GHz}$ and $f_{2}=16 \mathrm{GHz}$, hence $\Delta f=f_{2}-f_{1}=2 \mathrm{GHz}$. Based on sec. II A, seven different frequencies are produced by the multiplier and sent to the plasma. In this example, these frequencies are 84, 86, 88, 90, 92, 94 and $96 \mathrm{GHz}$. These frequencies are mixed with a local oscillator (LO) frequency, generated by a synthesizer followed by a $\times 6$-multiplier. The LO frequency is $f_{\mathrm{LO}}=\left(f_{1}+f_{2}\right) / 2+0.025 \mathrm{GHz}$, here $f_{\mathrm{LO}}=15.025 \mathrm{GHz}$. After downconversion of the probing frequencies with $f_{\mathrm{LO}}$, the resulting frequencies are $0.15 \mathrm{GHz}$ and $n \Delta f \pm 0.15 \mathrm{GHz}$, with $\mathrm{n}=1,2,3$. These can be downconverted to lower frequencies by mixing with the signal from three additional synthesizers at $f_{\mathrm{D} n}=n \Delta f+0.02 \mathrm{GHz}$. Since these synthesizers operate at lower frequencies than the synthesizers in the emission chain and the LO synthesizer, they can be substantially cheaper. After this downconversion, the resulting signals can be fed to IQ-detectors. In the present design in fig. 3, these IQ-detectors would require reference frequencies of 130, 150 and $170 \mathrm{MHz}$, which is easily affordable.

The advantage of such a detection system is that it can operate with comb reflectometer systems that use arbitrary frequencies. The only restriction is that the difference between individual comb frequencies has to be fixed. However, this is given by both generation concepts from secs. II A and II B. A drawback is that this system requires various synthesizers, which are comparably expensive. While a synthesizer providing frequencies to $20 \mathrm{GHz}$ at fixed output power is comparably affordable $(\sim 6 \mathrm{k} €)$, the synthesizers necessary for the two-tone input require output power control, which can bring the price up to $20 \mathrm{k} €$ each.

\section{PROOF OF CONCEPT}

Before deployment on AUG and the procurement of all final components, tests were performed in order to assure that the principle in sec. II actually works. To this end, both laboratory tests and tests on an AUG plasma confirmed the generation of frequency combs as layed out before. The laboratory tests were done with a spectrum analyzer, and the output spectra after the $\times 6$-multiplier were analyzed. The hardware components used were QuickSyn(C) FSW-0020 synthesizers, a Virginia Diodes(C) WR10AMI-I multiplier, and a Sage(C) SBP7531141015-1010-E1 $10 \mathrm{~dB}$ power amplifier. For the AUG plasma test, a V-band $(50-75 \mathrm{GHz})$ system was used in twotone input configuration (cf sec. II A).

\section{A. Laboratory Test of Two-tone Input}

Figure 4 shows spectra of two different frequency pairs applied to the $\times 6$-multiplier. The power has been measured behind $15 \mathrm{~cm}$ of WR-10 waveguide, the through-port of a $-10 \mathrm{~dB}$ coupler and an isolator. In consequence, real values are about $3 \mathrm{dBm}$ higher. Focusing first on the $15.8 / 17.0 \mathrm{GHz}$ pair, a comb of frequencies is obtained. The edge frequencies of the comb are then expected to be 94.8 and $102.0 \mathrm{GHz}$. Indeed, these frequencies are obtained, and frequencies lower than 94.8 $\mathrm{GHz}$ and higher than $102.0 \mathrm{GHz}$ exhibit significantly less power. In contrast, the intermodulation products show comparably strong powers, and the seven desired comb frequencies are within a range of $7 \mathrm{~dB}$. This is a good result and should yield reliable Doppler spectra for all the comb frequencies employed.

In contrast, using a different combination of input frequencies $(13.0 / 14.2 \mathrm{GHz})$, the specrum after the $\times 6$-multiplier is significantly more peaked, i.e. only three out of the seven desired frequencies are inside of a $10 \mathrm{dBc}$ power window. The remaining frequencies are at significantly lower input powers $(<-15 \mathrm{dBc})$, which is probably due to nonoptimized input power levels and non-optimum components involved. If this configuration was used in the experiment, this would mean that the low-power frequencies might be difficult to detect, or at least have insufficient signalto-noise ratios. However, it should be pointed out that these are first tests, and further optimizations are ongoing in order to improve the resulting spectra.

In summary, frequency comb generation via two-tone input works, and a test system can be set up comparably easily. However, there is significant work in the optimization of different frequency inputs into the multiplier. It is likely that a lookup table has to be constructed with optimum input power levels for each combination of frequencies foreseen to be used in the final system.

\section{B. Laboratory Test of Three-tone Input}

The method of three-tone input into the $\times 6$-multiplier has also been investigated in the laboratory. The DSB-mixer used 

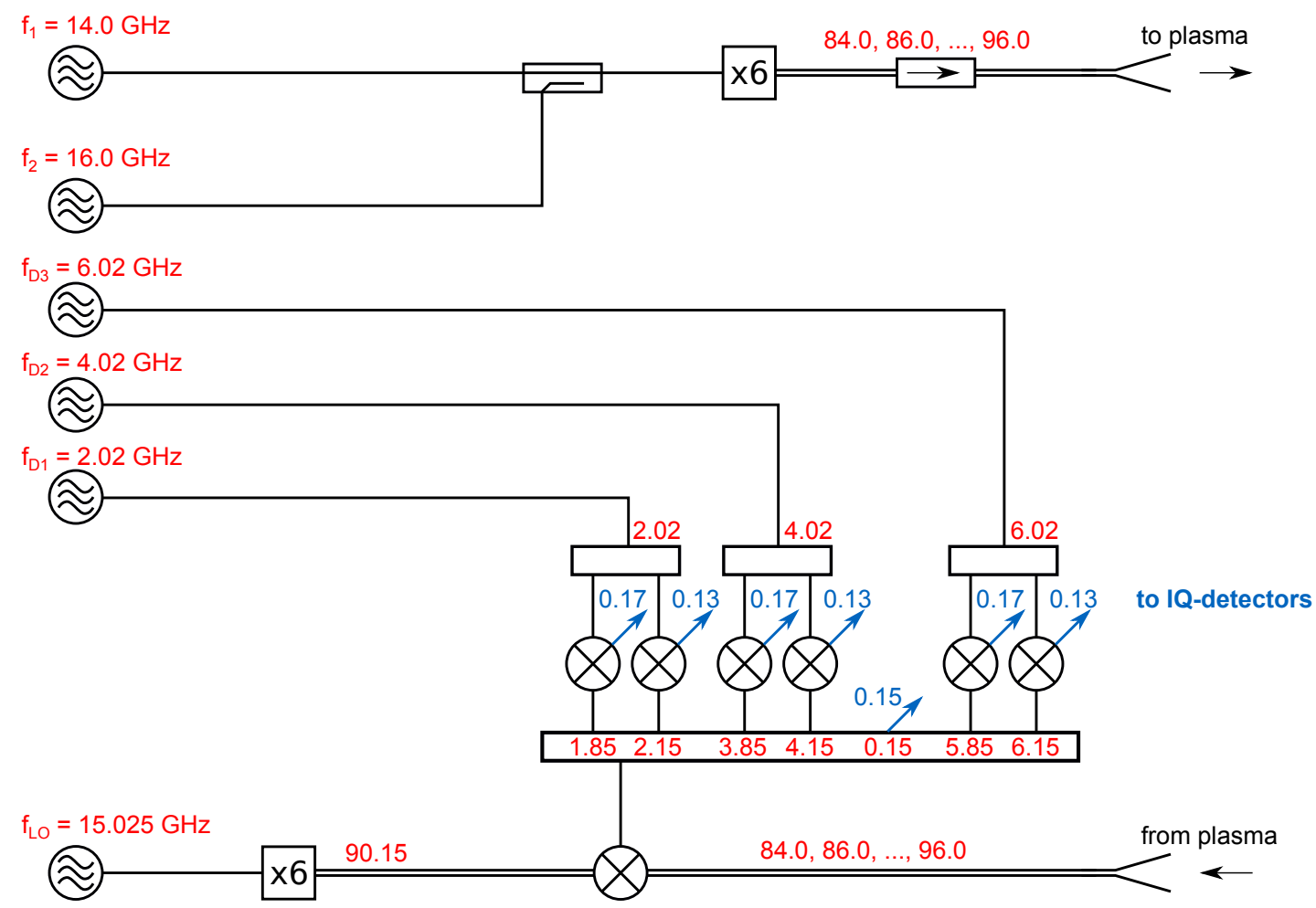

FIG. 3: Simplified microwave circuit diagram for the AUG comb Doppler reflectometer system. For simplicity, no amplifiers or attenuators are shown. The two-tone emission part is indicated in the upper part of the figure, while the detection component is shown in the lower part.

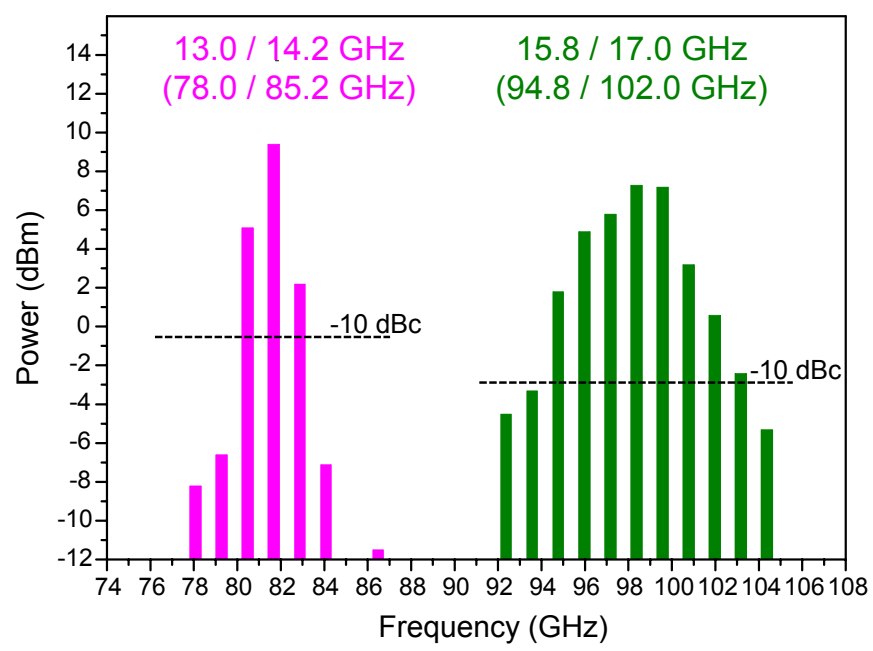

FIG. 4: Test of two-tone input into a $\times 6$-multiplier. The number of exploitable comb frequencies depends on the input frequencies, their power levels, etc. The power has been measured behind $15 \mathrm{~cm}$ of WR-10 waveguide, the through-port of a $-10 \mathrm{~dB}$ coupler and an isolator.

was a Marki(c) MMIQ-0520L model. For double sideband generation, the LO port and only one of the two in-phase and quadrature ports (with similar results) was used. First results are shown in fig. 5. In this example, $f_{\mathrm{LO}}=15.2 \mathrm{GHz}$. In (a), the intermediate frequency is $f_{\mathrm{IF}}=4.0 \mathrm{GHz}$. The resulting frequencies in the W-band have comparable power levels, which will give good signal-to-noise ratios once the system is deployed for operation on AUG. The measurement setup was as in sec. III A, so real values are about $3 \mathrm{dBm}$ higher. The central frequency, $91.2 \mathrm{GHz}$, is somewhat lower than the other comb frequencies. This result is obtained when the multiplier output is adjusted such that the $10 \mathrm{~dB}$ power amplifier is in mild saturation. Six frequencies are within $6 \mathrm{~dB}$, which is a good result for a comb reflectometer with variable center frequency and variable frequency separation. In fig. 5(b), the frequency difference is $f_{\mathrm{IF}}=6.0 \mathrm{GHz}$. In this case, only four frequencies lie within $6 \mathrm{~dB}$ power and the frequency at 109.2 $\mathrm{GHz}$ is at a level that cannot be used for a Doppler backscattering experiment. Still, apart from the frequency at $109.2 \mathrm{GHz}$, a comb spanning the whole W-band is generated, which is a good confirmation of the results expected from sec. II B.

\section{Experimental Verification of Two-tone Input on an AUG Plasma}

A system working in the V-band of frequencies (50 $75 \mathrm{GHz}$ ) is used to test the reliable operation of two-tone input into the multiplier. Since for the present V-band system [15] a $\times 4$-multiplier is used, a comb with five frequencies is expected following the argument in sec. II A. For the present test, two synthesizers were used to generate the $\times 4$-multiplier input at $f_{1}=15.25 \mathrm{GHz}$ and $f_{2}=16.25 \mathrm{GHz}$. After the multiplier, a comb is expected with the frequencies 61, 62, 63, 64 


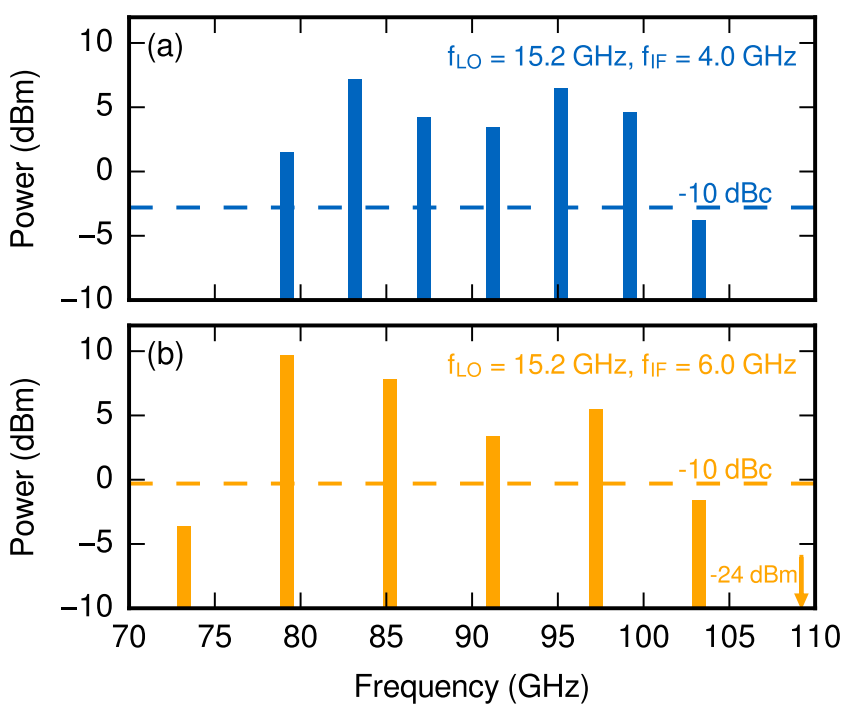

FIG. 5: Test of three-tone input into a $\times 6$-multiplier with central frequency $f_{\mathrm{LO}}=15.2 \mathrm{GHz}$. (a) $f_{\mathrm{IF}}=4.0 \mathrm{GHz}$ : six frequencies are within a power range of $6 \mathrm{~dB}$. (b) $f_{\mathrm{IF}}=6.0 \mathrm{GHz}$ : only four frequencies are within a power range of $6 \mathrm{~dB}$. Still, the comb spans the whole W-band. For both (a) and (b), the power has been measured behind $15 \mathrm{~cm}$ of WR-10 waveguide, the through-port of a $-10 \mathrm{~dB}$ coupler and an isolator.

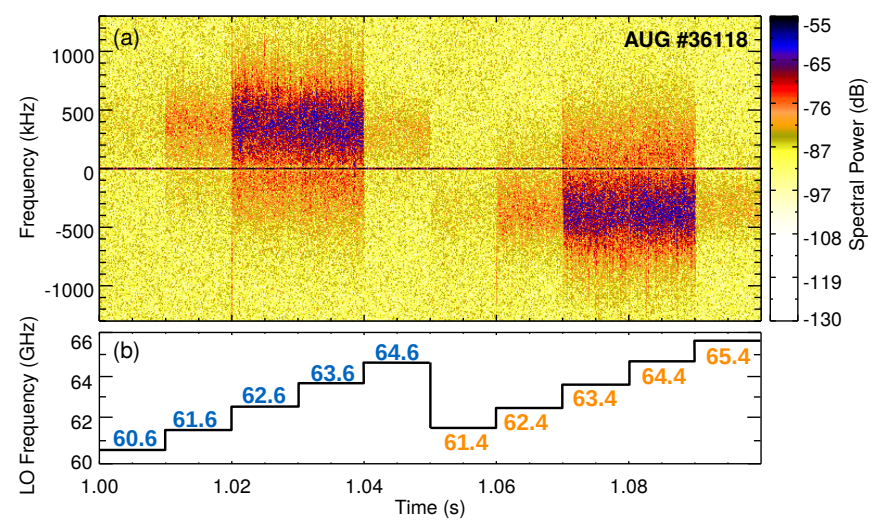

FIG. 6: Test of two-tone input on a plasma in the ASDEX Upgrade tokamak. The spectrogram of a $100 \mathrm{~ms}$ time sequence, where different probing frequencies are analyzed by stepping the local oscillator frequency (b). For details, refer to the text.

and $65 \mathrm{GHz}$. These frequencies are sent to the plasma, and the backscattered wave is analyzed. Since no dedicated system was available to detect a comb of frequencies, the LO frequency was stepped in $1 \mathrm{GHz}$ steps from 60.6 to $64.6 \mathrm{GHz}$, and then from 61.4 to $65.4 \mathrm{GHz}$. The two different patterns should result in the same backscattered spectra, but with the frequency axis reversed.

A spectrogram of this setup is shown for a $100 \mathrm{~ms}$ time window of AUG discharge \#36118, which was in L-mode during this time window, in fig. 6(a). The LO frequency is stepped every $10 \mathrm{~ms}$, which results in the frequency pattern shown in fig. 6(b). There is a Doppler shift visible for ev- ery probing frequency used. However, the strongest backscattered power is observed for the central frequencies, i.e. 63 and $64 \mathrm{GHz}$. The backscattered intensity at 62 and $65 \mathrm{GHz}$ is significantly lower, and for $61 \mathrm{GHz}$, while visible, the backscattered power is very low. This is because the central frequencies are stronger when using two-tone input than the ones corresponding to the edges of the generated comb spectrum. This is also visible in fig. 2, where the peaks at 84 and $96 \mathrm{GHz}$ are smaller than those at 88,90 and $92 \mathrm{GHz}$. In the test with plasma, this effect is more significant. However, it should be pointed out that the frequency comb in this test was generated with two synthesizers without output power control. It is highly likely that the power distribution amongst the different probing frequencies can be balanced much better than is the case in fig. 6(a). Another observation is that the Doppler shift goes from positive to negative frequencies when the intermediate frequency (IF) is changed from negative $(-400 \mathrm{MHz})$ to positive $(+400 \mathrm{MHz})$, which is to be expected, and a satisfying confirmation of the working principle of the two-tone input system.

The spectra of the backscattered wave are presented in fig. 7 for the comb of five different probing frequencies. The blue spectra have been obtained by using the negative IF, and the orange spectra have been obtained using a positive IF, and have then been flipped. The spectra compare very well. The power drop from the strongest backscattered power $(-60 \mathrm{~dB}$ in fig. $7(\mathrm{~d}))$ to the one with lowest backscattered power $(-80 \mathrm{~dB}$ in fig. $7(\mathrm{a}))$ is about $20 \mathrm{~dB}$. As mentioned before, this can likely be at least partly avoided by using adequate mulitplier input power levels in order to obtain a frequency comb of comparable power.

This result shows that a comb generation via the two-tone input method is possible, even without any optimizations in terms of $\times 4$-multiplier input power, adaptation of power levels in the detection branch, or any filtering. Hence, the system is now being put forward, and it is expected to be operational in summer 2020 for deployment on AUG.

\section{SUMMARY}

The design of a new comb reflectometer system for the ASDEX Upgrade tokamak has been presented. The system will work in the W-band of frequencies, and the comb will be generated either by two-tone or by three-tone input into a $\times 6$ multiplier. The detection system is based on a two-step downconversion, which will provide the intermediate frequencies to be fed into standard IQ-detectors. First laboratory tests and tests on an AUG plasma have shown the reliability of the concept.

The advantages of the presented concepts are that it can work at arbitrary frequencies and arbitrary frequency separations. Only the frequency separation between any two neighboring frequencies has to be the same. This will allow studies of the perpendicular velocity and its shear at various radial positions in the plasma simultaneously, radial propagation of events or the radial correlation measurement and its dynamic behavior. 

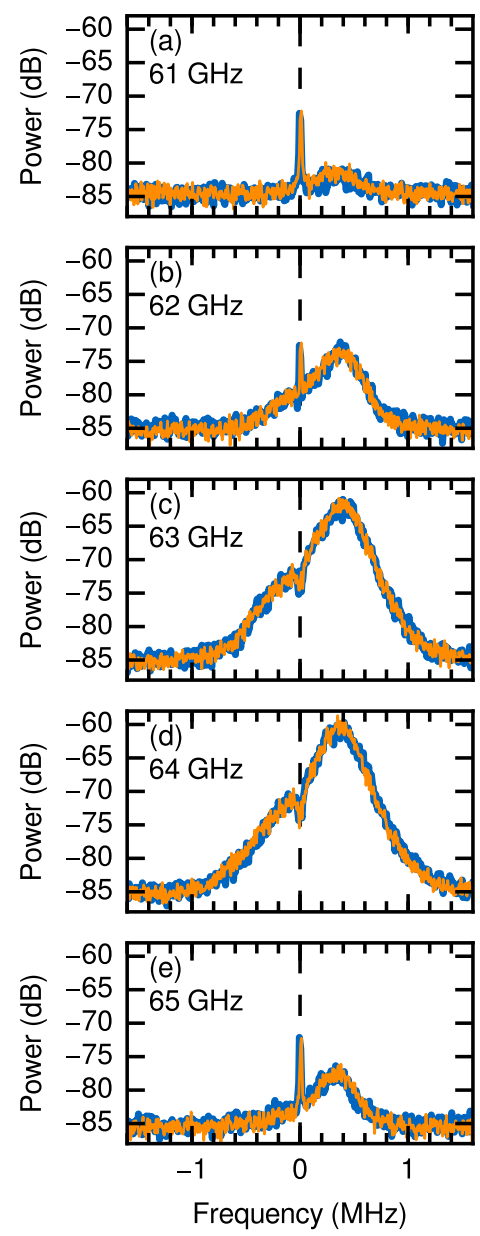

FIG. 7: Spectra obtained from a system based on two-tone input into the multiplier from the AUG tokamak. The center frequencies show the largest power $(-60 \mathrm{~dB}$ in (c) and (d)). Although the power is significantly lower at 61 and $65 \mathrm{GHz}$, a Doppler shifted peak is still visible. For details refer to the text.

\section{Acknowledgments}

This work has been carried out within the framework of the EUROfusion Consortium and has received funding from the Euratom research and training programme 2014-2018 and 2019-2020 under grant agreement No 633053. The views and opinions expressed herein do not necessarily reflect those of the European Commission.
[1] H. J. Hartfuss et al., Rev. Sci. Instrum. 65, 2284 (1994).

[2] L. Cupido, J. Sanchez, and T. Estrada, Rev. Sci. Instrum. 75, 3865 (2004).

[3] N. Oyama et al., Plasma Fus. Res. 6, 1402014 (2011).

[4] J. C. Hillesheim et al., Rev. Sci. Instrum. 80, 083507 (2009).

[5] W. A. Peebles et al., Rev. Sci. Instrum. 81, 10 D902 (2010).

[6] N. A. Crocker et al., Plasma Phys. Control. Fusion 53, 105001 (2011).

[7] T. Tokuzawa et al., Plasma Fus. Res. 9, 1402149 (2014).

[8] Z. Shi et al., Rev. Sci. Instrum. 87, 113501 (2016).
[9] R. Soga et al., Journal of Instrumentation 11, C02009 (2016).

[10] J. Q. Hu et al., Rev. Sci. Instrum. 88, 073504 (2017).

[11] T. Tokuzawa et al., Rev. Sci. Instrum. 89, 10H118 (2018).

[12] P. Molina Cabrera et al., Rev. Sci. Instrum. 89, 083503 (2018).

[13] M. Y. Wang et al., Rev. Sci. Instrum. 89, 093501 (2018).

[14] X. Feng et al., Rev. Sci. Instrum. 90, 024704 (2019).

[15] R. Sabot, P. Hennequin, and L. Colas, Fusion Sci. Technol. 56, 1253 (2009). 